\section{$\underset{\substack{\text { hommes } \\ \text { \& migrations }}}{ }$}

\section{Hommes \& migrations}

Revue française de référence sur les dynamiques

migratoires

1306 | 2014

Ecriture et migration

\title{
Agota Kristóf, une écriture de ruines aspirant à la survie
}

Décryptage de la figure du double dans Le Grand Cahier

\section{Melinda Mod}

\section{(2) OpenEdition}

Journals

Édition électronique

URL : http://journals.openedition.org/hommesmigrations/2803

DOI : 10.4000/hommesmigrations.2803

ISSN : 2262-3353

Éditeur

Musée national de l'histoire de l'immigration

Édition imprimée

Date de publication : 1 avril 2014

Pagination : 67-70

ISBN : 978-2-919040-27-8

ISSN : 1142-852X

Référence électronique

Melinda Mod, "Agota Kristóf, une écriture de ruines aspirant à la survie », Hommes \& migrations [En

ligne], 1306 | 2014, mis en ligne le 01 avril 2017, consulté le 10 décembre 2020. URL : http://

journals.openedition.org/hommesmigrations/2803; DOI : https://doi.org/10.4000/

hommesmigrations.2803 


\section{AGOTA KRISTÓF, UNE ÉCRITURE DE RUINES ASPIRANT À LA SURVIE DÉCRYPTAGE DE LA FIGURE DU DOUBLE DANS LE GRAND CAHIER}

par MELINDA MÓD, doctorante, université Paris-VIII, Centre d'études féminines et d'études de genre (CNRS-CRESPPA-GTM).

La mise en scène d'une identité morcelée par le traumatisme de l'exil traverse l'œuvre de l'écrivaine d'origine hongroise Agota Kristóf. L'étude de l'emploi systématique du pronom "nous" dans Le Grand Cahier, pour articuler I'histoire de ses personnages jumeaux, permet de caractériser les modalités du dédoublement d'une identité douloureuse. L'écriture d'Agota Kritóf porte l'urgence d'une réunification qui ne peut avoir lieu que dans et par la pratique littéraire.

L’année 1956 marquera à jamais l'histoire de la Hongrie. Après le déclenchement de la révolution spontanée contre l'occupation soviétique du territoire hongrois, 200000 citoyens fuient le pays et s'installent en exil ${ }^{1}$. L'un d'entre eux est Agota Kristóf ${ }^{2}$, jeune mère de 21 ans à l'époque. Comme pour de nombreux exilés, il ne s'agit pas d'une décision délibérée : c'est dans l'urgence qu'elle suit son mari, impliqué dans la politique et craignant des représailles. Par le hasard du destin, c'est en Suisse romande qu'ils trouvent refuge. Née en 1935 à Csikvánd, un petit village situé près de la frontière austro-hongroise, Kristóf a été témoin de l'occupation de son pays natal d'abord par les Allemands, puis par les Russes à la fin de la Seconde Guerre mondiale. L'environnement de l'enfance, la proximité de la frontière, l'interaction des langues, la ville de Köszeg, où elle a habité dès l'âge de 9 ans, deviendront des topos circulaires dans son écriture. L'expérience traumatique d'avoir quitté son pays et sa famille contre son gré, d'avoir perdu ses manuscrits lyriques, sa langue et ses rêves tisseront ses récits que la critique qualifiera de "cruels" et "nihilistes". 


\section{Écriture et expérience traumatique de l'exil}

L'Analphabète, un recueil de textes à caractère autobiographique, rédigés entre 1989 et $1990^{3}$, ainsi que de nombreuses interviews éclairent le lien direct entre le vécu traumatique de l'exil de Kristóf et son style "inquiétant", "troublant" et provoquant un "sentiment de malaise" chez le lecteur ${ }^{4}$. Le succès de ses œuvres romanesques, théâtrales et de ses nouvelles, traduites en plus de quarante langues, nous incite à nous interroger sur sa manière de capter les résonances fines de la condition d'exilé. Estce son style, les thèmes, l'architecture de ses textes, le mélange des traditions littéraires ou encore le caractère insaisissable de la narration qui font remarquer sa production littéraire, au point qu'elle figure désormais au programme de littérature de l'enseignement français ${ }^{5}$ ? Cet article se focalisera en particulier sur les divers aspects de la mise en scène des jumeaux, dont l'usage du pronom personnel "nous" dans Le Grand Cahier. L'objectif est d'analyser le lien entre la mise en scène de la figure du double et l'écriture fiévreuse de l'exil.

\section{La Trilogie, une histoire des enfants sans enfance}

La Trilogieregroupelestexteslesplusconnus d'Agota Kristóf. Elle se compose du Grand Cahier (1986), de La Preuve (1988) et du Troisième Mensonge (1991)6. Une guerre, probablement la Seconde Guerre mondiale, fournit la toile de fond à l'intrigue du Grand Cahier. Les protagonistes gémellaires aux noms anagrammatiques, Claus et Lucas, s'y retrouvent isolés à l'extrémité du village $\mathrm{K}$. (faisant référence à Kőszeg), chez une femme en qui nous reconnaîtrons leur grand-mère supposée. Dans le contexte de la guerre et dans l'environnement de cette femme brute, personne ne leur manifeste la moindre affection. Pour reprendre la remarque de Carine Trevisan, les jumeaux vivent en dehors du cadre habituel de l'enfance, se trouvent "expulsé[s] de [leur] position d'enfant ${ }^{7 "}$. "Grand-Mère nous frappe souvent, avec ses mains osseuses, avec un balai ou un torchon mouillé. (...) D'autres gens nous donnent aussi des gifles et des coups de pied, nous ne savons même pas pourquoi. (...) Nous décidons d'endurcir notre corps pour pouvoir supporter la douleur sans pleurer. (...) Au bout d'un certain temps, nous ne sentons effectivement plus rien.".

Les jumeaux, afin de survivre dans leur solitude et de s'habituer aux diverses cruautés de la vie, sont prêts à effectuer des actes qui relèvent du domaine de la violence, de la brutalité, de la triste insensibilité. Ils s'inventent des exercices d'endurcissement, de jeûne, de mendicité 9 . Tuer des animaux ou leur père, accrocher le squelette de leur mère et de leur sœur dans leur chambre, voilà ce qui fait partie de leur quotidien d'où disparaît tout sentiment.

\section{L'apparition de la figure du double par le "nous" unificateur}

L'intrigue du Grand Cahier, comme son titre l'indique, s'établit par la rédaction d'un cahier d'écolier. C'est dans ce livret que les jumeaux notent tout ce qui arrive autour d'eux, suivant strictement la seule règle qu'ils ont définie eux-mêmes, notamment celle de purifier le texte, d'en évacuer tout sentiment, tout adjectif, toute subjectivité ${ }^{10}$. Bien que les narrateurs de ces histoires soient les deux frères,

3. Agota Kristóf, L’Analphabète, Carouge-Genève, Zoé, 2004. 4. Carine Trevisan, "Les enfants de la guerre : Le Grand Cahier d’Agota Kristóf", in Amnis, juin 2006. 5. John Kristian Sanaker, "Le Grand Cahier d’Agota Kristóf - une singularité francophone”, in Romansk Forum, vol. 2, n 16, 2002. 6. Agota Kristóf, Le Grand Cahier, Paris, Seuil, 1986 ; La Preuve, Paris, Seuil, 1988 ; Le Troisième Mensonge, Paris, Seuil, 1991. 7. Carine Trevisan, op.cit. 8. Agota Kristóf, Le Grand Cahier, Paris, Seuil, 1986, pp. 20-21. 9. "Az út Csikvándtól Kínáig”, entretien réalisé par András Petöcz le 22 octobre 2009, in Élet és irodalom, $n^{\circ}$ 43. Il est intéressant de noter que Kristóf puise dans sa propre enfance pour décrire ces scènes d'endurcissement. Elle explique dans l'interview réalisée en hongrois : "Je ne me souviens plus très bien, mais je sais que nous imaginions que l'un de nous était riche, l'autre pauvre, puis des fois nous parlions en chantant pendant des heures, et, c'était déjà au collège, nous avons décidé avec une de mes amies de ne pas manger, c'est-à-dire, nous avons fait un exercice de jeûne." 10. L'écrivaine explique qu'elle suit les mêmes règles dans sa pratique textuelle. "Egy érzelmeskedés nélküli élet. Beszélgetés Agota Kristof írón vel”, propos recueillis par Zsigmond Papp Sándor, in Népszabadság, 6 octobre 2009. 
le pronom "je" est curieusement absent de l'intégralité du premier volet de la Trilogie. À sa place, le lecteur découvre l'usage permanent de "nous", dont il est possible de relever la fonction unificatrice.

Le premier constat des théories identitaires postule que la possibilité de tout dialogue s'enracine dans une relation éminemment asymétrique ${ }^{11}$. Suivant la définition de l'altérité proposée par Martin Buber (notamment que "la rencontre me place en face de l'autre ${ }^{12 \text { ") }}$, on note que Kristóf écarte la possibilité même de l'altérité entre les jumeaux. En employant uniquement la première personne du pluriel dans la narration, et cela également dans des situations où il est virtuellement impossible que deux personnes distinctes agissent symétriquement et qu'ils aient les mêmes sentiments, elle opte pour la construction d'un "nous" ayant la valeur d'un "nous" idéal. Il est probable que des phrases du type "Nous ne sortons jamais l'un sans l'autre. Nous allons partout ensemble 13 " ou "nous

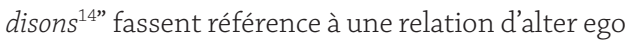
idéale où l'asymétrie entre les frères n'existe pas.

\section{L'usage des pronoms sème le doute}

Selon l'idée de l'absence d'altérité, le lecteur peut supposer que la narration se fait par une seule voix dédoublée, marquée grammaticalement par le pronom "nous", et non par deux énonciateurs distincts et pourtant semblables en tout point. Des indices relatifs à l'unité des deux corps sembleraient témoigner de l'invention d'un double mental par le personnage narrateur, soulevant le voile de la possibilité d'une éventuelle schizophrénie. La transcription d'un dialogue avec un policier qui prend place après un interrogatoire propose une fausse vue d'extérieur sur les jumeaux. Les personnages et le narrateur s'entremêlent dans un seul référent dont la vue, la bouche, la tête ne se portent que sur une singularité : "- Nous avons mal partout. Le plus léger mouvement nous fait retomber dans une semi-inconscience. Notre vue est voilée, nos oreilles bourdonnent, notre tête résonne. Nous avons terriblement soif. Notre bouche est sèche. - Des heures passent ainsi. Nous ne parlons pas. Plus tard, un policier entre, il nous demande : - Vous avez besoin de quelque chose $^{15}$ ?" La situation narrative se complique par la construction anagrammatique des prénoms Lucas et Claus. Kristóf met en place une écriture circulaire où l'histoire s'affiche par des bribes du cahier d'écolier et où les deux narrateurs supposés convergent vers un seul référent. Valérie Petitepierre souligne judicieusement que le "nous" de la narration du Grand Cahier ne correspond pas à la définition proposée par Benveniste, qui regroupe un "je" et un non "je"16. Chez Kristóf, le "nous" se réfère à l'unité symbiotique parfaite de deux corps et de deux identités identiques. Le "nous" s'y situerait sur le plan de deux "je" additionnés, établissant une pathologie schizoïde. Michèle Bacholle relève également qu' "en fait, plus que d'une symbiose, il semblerait s'agir d'une fusion pure et simple. En effet, à divers moments le lecteur a l'impression qu'il pourrait bien n'y avoir qu'un seul personnage $e^{17 "}$.

\section{Une modification de la forme du texte}

Une théorie du "nous" fusionnel se trouve justifiée également par l'absence de tout dialogue réel entre les deux protagonistes. Les rares moments de discussion entre les deux frères tournent au dialogue absurde, les réponses ne correspondant pas aux questions. Les deux frères jouent au jeu des sourds et des aveugles, un jeu qui symbolise l'exclusion de toute forme de réciprocité : "L'un de nous fait l'aveugle, l'autre fait le sourd. (...) L'aveugle parle lentement pour que le sourd puisse lire sur ses lèvres : - J'entends les avions. (...) Sinon, tout est silencieux. Le sourd lit sur les lèvres de l'aveugle et répond: - Oui, la rue est vide ${ }^{18}$." Suivant l'idée de Cécile Kovacshazy que "le double serait relégué au statut du thème s'il ne modifiait en profondeur la forme du texte ${ }^{19 "}$, on pourrait 
concevoir l'écriture chez Kristóf comme un endroit unificateur fantasmatique, comme un espace de réconciliation, face aux coupures temporelles et géographiques de l'exil. Par l'écriture se mettent en place des stratégies où "s'endure le processus interminable, indéfiniment phantasmatique, de lidentification ${ }^{20 "}$ "d'une "identité jamais donnée, reçue ou atteinte ${ }^{21 "}$. C'est par le geste de l'écriture que le narrateur matérialise un univers dans lequel les deux frères auraient toujours vécu ensemble, ce qui ne fut pas le cas dans la réalité : le lecteur le saura au fil des pages de La Preuve et du Troisième Mensonge.

\section{L'identité troublée des exilés}

Des personnages troublés, violents et solitaires emplissent la Trilogie. Par leur figure, s'exprime la douleur de la séparation avec un pays, une langue, une famille. Tijana Miletić dit de manière pertinente qu'il n'est pas étonnant que le thème des doubles apparaisse fréquemment dans les écrits d’immigrés, cela peut être considéré comme un moyen approprié pour visualiser l'identité troublée et parfois déchirée de ceux qui traitent deux langages différents comme leur langue maternelle22." La scène clôturant Le Grand Cahier confronte le lecteur à l'image du double dans un aspect nouveau par rapport à ce que le pronom "nous" évoquait jusque-là. Il ne s'agit plus des particularités de l'usage morphosyntaxique, mais d'un véritable dédoublement des personnages au sens physique et symbolique du terme. Les deux frères ne voyant pas d'autre issue aux horreurs de la guerre décident que l'un d'eux traversera la frontière. Cet acte entraînera le changement de l'ordre du quotidien, de la langue et de la vision de soi et dirigera les deux tomes suivants de la Trilogie vers l'individualisation de la voix de l'énonciateur. Chez Kristóf, "né de l'écriture ${ }^{23 ", ~ l e ~}$ double n'y retourne pas. La fuite de l'autre identique "nous sert pour nous situer, il est notre repère et, négativement, notre véritée ${ }^{24 "}$, souligne Wladimir Troubetzkoy, qui ajoute une note pertinente en rapport avec l'analyse de la scène de séparation des jumeaux. Il explique notamment que "le Double est un évadé en cavale, il est ce qui de nous erre aux alentours de nous ${ }^{25}$. Cet aspect du thème littéraire des jumeaux amène le lecteur à considérer le personnage Claus-Lucas au-delà de son rôle de narrateur. Le double dépassera alors cette fonction dans l'ordre de l'énonciation pour incarner la métaphore littéraire par excellence de l'altérité de soi.

$\boldsymbol{\Gamma}$

\section{Écrire l'indicible traumatisme de la séparation}

À travers Le Grand Cahier, il est possible de découvrir une double analogie dans la figure du double : la superposition de deux référents en un seul et la perte du référent clair. Chez Kristóf, cette perte "est compensée par une quête qui a pour objet le récit lui-même ${ }^{26 "}$. L'écrivaine hongroise a saisi l'indicible traumatisme de la séparation par la création d'un espace discursif monstrueux et fiévreux, dans lequel se produit l'aliénation de soi, la cruauté inimaginable remplace les jeux d'enfants, où chaque personnage reste étranger à l'autre. L'écriture s'y transforme en urgence, en une "écriture suicidaire ${ }^{27 "}$, par laquelle Kristóf crée un espace où elle ne vit plus la vraie vie, mais "ce qu'on écrit ${ }^{28 ”}$. Cette explication de la force performative de l'écriture fait appel à ce que Julia Kristeva note dans sa fort belle phrase: "L'étranger est un rêveur qui fait l'amour avec l'absence ${ }^{29 "}$. L'absence et l'étrangeté non irrémédiable monopolisent l'espace narratif, que seul l'acte de passer la frontière, partir en exil, réussit à faire évoluer. 